\title{
EDITORIAL
}

\section{Design Faults}

One of the most disturbing reasons for rejecting a paper submitted to the Journal, from my point of view, is when the editorial report indicates that there is a flaw in the design of the study which is so fundamental that no conclusions can be drawn from the paper, certainly none that the author would wish to draw.

When composing the letter of rejection I try to think how best to 'let the author down gently' by trying to make some positive suggestions. Often the only practical suggestion I can make is to advise the authors to repeat the work using the editorial report as a basis for a new design and to regard the work for the existing paper as a learning exercise. However, I am well aware that, in the current climate of research support in many countries, it is often not possible to repeat the work because the person responsible has moved on or the project funding for the work in question has been exhausted. In the 'good old days' one could often find a way of repeating all or part of a piece of work so that all was not lost with regard to publication. This, of course, assumed that there were some interesting findings to salvage from the rejected paper. I know that many authors who can find no additional resources for repeating the work and who are under pressure to publish something will simply send the paper to another journal.

Others, quite properly, will argue that the design is not flawed and our editorial report is based on a false argument or has missed the point of the work. Although this response involves me and our editorial team in some very hard thinking, I welcome this type of response because I know from personal experience that editors are not infallible (even if their reports sometimes give the impression that they are) and that everyone can learn from having to respond to criticism. As someone who sees the role of the Journal to advance nutritional science, I do not wish to 'lose' any paper that really does contain some original contribution to the nutritional sciences.

Nevertheless, I think that the rejection of papers due to design faults raises more serious issues because it implies that the resources for research are being, to put it bluntly, wasted. This in itself is bad enough but such a rejection often implies that experimental animals have been used unnecessarily or that human subjects have had their time and commitment used to no good scientific purpose.

You may say that just because the $B J N$ editors do not like a design the paper is unpublishable; another editor will be either more perceptive or less demanding and the paper will appear in print eventually.

Where peer reviewers have genuine and justifiable criticism that a design is fatally flawed should we as nutritional scientists really be content with hawking a paper around? I think not: the real answer is the recognition of the fundamental importance of experimental design. I had one author who when challenged about the design of a study said 'there was no design because it was an observational study', an answer that I did not find convincing. As I have said before, the actual observations that one chooses to make in an observational study are based on some hypothesis regarding the factors that may be important and that the study does therefore have a design de facto to test that hypothesis. I know, because we see the papers, that some authors work on the principle that we will measure everything that we can think of and then wheel out our latest statistical package to sort out what is relevant or, more correctly, what will achieve statistical significance. Such an approach is 
also a waste of resources and neglects some of the basic principles of scientific logic. It also often places the statistician in the unenviable position of having to analyse data where the experimental measurements have been made without thought of the analyses to be applied. In most cases, however, rejections because of faulty design are not made on statistical grounds alone because statisticians are frequently required, by those who should know better, to analyse data from a design in which he or she has had no prior say. This means that statistical faults in design may often be correctable or at least a method can be found to salvage usable data. Design faults of a more fatal nature stem from the lack of either a sound and well thought out hypothesis or from setting up a series of experimental treatments only to find that the treatments did not conform to the design: for example, the differences between the diets were much smaller than planned so the effects of the treatments were not different; or comparing two fibre sources supposedly because of differences in their fermentability without checking that they did differ.

Achieving a sound design depends on setting up a working hypothesis and deciding how that hypothesis is to be tested, preferably in consultation with a statistician. It is often useful to involve a 'devil's advocate' in such planning whose role is to ask 'what if' questions about the type of data that might result from the study. The practical details of the experiment need then to be considered. Pilot runs of preparing and characterizing diets before feeding them could have saved many authors from excusing findings that did not achieve significance.

One can only re-emphasize what is sometimes dismissed as a counsel of perfection that the statistical input should come at the design stage not as a 'long-stop' to retrieve any usable data from a study. However, the central feature of good design is a clear idea or hypothesis of what the study is intended to do. A testable hypothesis gives the statistician a clear idea of what the experimental study is about and how to design it to address those ends. I would also make a plea that the hypothesis is examined for its logical feasibility first because a paper we discussed recently at a journal club here in Wageningen had a patently falsifiable hypothesis, on the basis of the literature cited, without the need for any experimental work at all.

D. A. T. Southgate 